\title{
On biembeddings of Latin squares
}

\author{
M. J. Grannell, T. S. Griggs \\ Department of Mathematics and Statistics \\ The Open University \\ Walton Hall \\ Milton Keynes MK7 6AA \\ United Kingdom
}

\author{
M. Knor \\ Department of Mathematics \\ Faculty of Civil Engineering \\ Slovak University of Technology \\ Radlinského 11 \\ 81368 Bratislava \\ Slovakia
}

Submitted: Sep 7, 2008; Accepted: Aug 10, 2009; Published: Aug 21, 2009

Mathematics Subject Classifications: 05B15, 05C10

\begin{abstract}
A known construction for face 2-colourable triangular embeddings of complete regular tripartite graphs is re-examined from the viewpoint of the underlying Latin squares. This facilitates biembeddings of a wide variety of Latin squares, including those formed from the Cayley tables of the elementary Abelian 2-groups $C_{2}^{k}(k \neq 2)$. In turn, these biembeddings enable us to increase the best known lower bound for the number of face 2-colourable triangular embeddings of $K_{n, n, n}$ for an infinite class of values of $n$.
\end{abstract}

\section{Background}

In [6] a recursive construction was presented for face 2-colourable triangular embeddings of complete tripartite graphs $K_{n, n, n}$. The construction was used in that paper to provide lower bounds of the form $2^{a n^{2}}$ for the numbers of face 2-colourable triangular embeddings of both complete tripartite graphs $K_{n, n, n}$ and complete graphs $K_{n}$, for certain values of $n$. In a subsequent paper [2], a generalization of this construction was used to increase these lower bounds to ones of the form $n^{a n^{2}}$ for certain values of $n$. A face 2-colourable triangular embedding of $K_{n, n, n}$ corresponds to a biembedding of two Latin squares. The purpose of this current paper is to re-examine the construction from [6] from the viewpoint of the Latin squares involved. This alternative focus enables us to obtain new results about biembeddings of Latin squares and to improve the bound given in [2].

For general background material on topological embeddings, we refer the reader to [7] and [9]. Our embeddings will always be in closed connected 2-manifolds without a boundary. A graph embedding is face 2-colourable if the faces may be coloured in such a way that any two faces with a common boundary receive different colours. We will always 
take the colours to be black and white. It was shown in [3] that a triangular embedding of $K_{n, n, n}$ is face 2-colourable if and only if the supporting surface is orientable, and the surface is therefore a sphere with an appropriate number of handles.

A face 2-colourable triangular embedding of $K_{n, n, n}$ determines two transversal designs, $\operatorname{TD}(3, n)$, one for each colour class. Such a design comprises an ordered triple $(V, \mathcal{G}, \mathcal{B})$, where $V$ is a $3 n$-element set (the points), $\mathcal{G}$ is a partition of $V$ into three disjoint sets (the groups) each of cardinality $n$, and $\mathcal{B}$ is a set of 3-element subsets of $V$ (the triples), such that every unordered pair of elements from $V$ is either contained in precisely one triple or one group, but not both. The vertices of the embedded graph $K_{n, n, n}$ form the points of each design, the tripartition determines the groups, and the faces in each colour class form the triples of each design.

The connection with Latin squares is that a $\operatorname{TD}(3, n)$ determines a Latin square of order $n$ by assigning the three groups of the design as labels for the rows, columns and entries (in any one of six possible orders) of the Latin square. Conversely any Latin square of order $n$ determines a $\operatorname{TD}(3, n)$. Two Latin squares are said to be in the same main class or paratopic if the corresponding $\operatorname{TD}(3, n)$ s are isomorphic. Thus a face 2 -colourable triangular embedding of $K_{n, n, n}$ may be considered as a biembedding of two $\operatorname{TD}(3, n) \mathrm{s}$ or, equivalently, two Latin squares. To be precise, we say that two Latin squares of order $n$ are biembeddable in a surface if there is a face 2-colourable triangular embedding of $K_{n, n, n}$ in which the face sets forming the two colour classes give paratopic copies of the two squares.

Given a Latin square $L$ of side $n$, we may use the notation $k=L(i, j)$ to denote that entry $k$ appears in row $i$ column $j$ of $L$; alternatively we may write $(i, j, k) \in L$. In this latter form, the triples of any Latin square will always be specified in (row, column, entry) order. Note however that in a biembedding of two Latin squares, the vertices of faces from one colour class will appear clockwise in the cyclic order (row, column, entry), while those from the other will appear anticlockwise if taken in the same cyclic order. A parallel class of triples in a $\operatorname{TD}(3, n)$ is a set of triples in which each point of the design appears precisely once. Such a parallel class is equivalent to a transversal in a corresponding Latin square.

In this paper we need to distinguish carefully between statements about biembeddings of Latin squares where paratopic copies are allowed, and statements about particular realizations of Latin squares. For Latin squares $A$ and $A^{\prime}$ with common sets of row labels, of column labels, and of entries, we will write $A \bowtie A^{\prime}$ (to be read as $A$ biembeds with $A^{\prime}$ without relabelling), if and only if the particular realizations of $A$ and $A^{\prime}$ form a surface embedding; that is to say that the triangles formed by the (row, column, entry) triples of $A$ and $A^{\prime}$ may be sewn together along their common edges to form the surface. With a slight abuse of notation we also use $A \bowtie A^{\prime}$ to denote the actual embedding itself.

It is known that there are many nonisomorphic biembeddings of Latin squares. However, up until now, the only clearly identifiable family of Latin squares that are known to admit biembeddings has been the family of cyclic squares $C_{n}$ defined by $C_{n}(i, j)=i+j$ $(\bmod n)$. In [5] it was proved that for many values of $n$, there are exponentially many mates $M_{n}^{\prime}$, all of which are paratopic to $C_{n}$ and are such that $C_{n} \bowtie M_{n}^{\prime}$. In the current 
paper, it is shown that a wide variety of Latin squares admit biembeddings; in particular $C_{2}^{k}$ for $k \neq 2$. Note however that, apart from the trivial case $k=1$, any mate $A_{k}^{\prime}$ of $C_{2}^{k}$ such that $C_{2}^{k} \bowtie A_{k}^{\prime}$ cannot be a paratopic copy of $C_{2}^{k}[8]$.

\section{Construction}

The construction to which we refer is taken from [6]. It produces a face 2-colourable triangular embedding of $K_{m n, m n, m n}$ from face 2-colourable triangular embeddings of $K_{m, m, m}$ and $K_{n, n, n}$, provided that each of the $K_{m, m, m}$ embeddings has a parallel class in one colour. We start by describing this construction, using a labelling of the vertices of the embedding that will help in the subsequent discussion.

So, suppose that for $0 \leqslant u \leqslant m-1, \phi_{u}$ is a face 2-colourable triangular embedding of $K_{n, n, n}$ with vertex set $R_{u} \cup C_{u} \cup E_{u}$, where $R_{u}=\left\{r_{n u+i}: 0 \leqslant i \leqslant n-1\right\}, C_{u}=$ $\left\{c_{n u+i}: 0 \leqslant i \leqslant n-1\right\}$ and $E_{u}=\left\{e_{n u+i}: 0 \leqslant i \leqslant n-1\right\}$ are three disjoint sets. We use the letters $r, c$ and $e$ because these will later be related to the row, column and entry labels of a Latin square. We also suppose that for each oriented white triangle $\left(r_{i}, c_{j}, e_{k}\right)$ $\left(=\left(r_{n 0+i}, c_{n 0+j}, e_{n 0+k}\right)\right)$ of $\phi_{0}$, the ordered triple $\left(r_{n u+i}, c_{n u+j}, e_{n u+k}\right)$ defines an oriented white triangle of $\phi_{u}$ for each $u \in\{0,1, \ldots, m-1\}$. In essence, this means that the embeddings $\phi_{u}$ all have the "same" white triangles with the same orientations, although the black triangles may be different. Then, for $0 \leqslant i, j \leqslant n-1$, let $\psi_{i, j}$ be a face 2colourable triangular embedding of $K_{m, m, m}$ having a parallel class of $m$ black triangular faces. Initially, the supporting surfaces of all the embeddings $\phi_{u}$ and $\psi_{i, j}$ are taken to be disjoint from one another.

Next list the $n^{2}$ white triangles of $\phi_{0}$; without loss of generality, we can take these as given by $W_{i, j}=\left(r_{i}, c_{j}, e_{k}\right)$, where $k$ is uniquely determined by $(i, j)$. Then label the $m$ black triangles forming the parallel class in $\psi_{i, j}$ as $\left(r_{n u+i}^{*}, c_{n u+j}^{*}, e_{n u+k}^{*}\right), 0 \leqslant u \leqslant$ $m-1$, in some order, taking care to respect the three vertex parts of the embedding so that one vertex part receives labels $r^{*}$, another $c^{*}$ and the third $e^{*}$. Then each vertex of $\psi_{i, j}$ is uniquely labelled and, for each oriented white triangle $\left(r_{n u+i}, c_{n u+j}, e_{n u+k}\right)$ of the embedding $\phi_{u}$, there is a corresponding black triangle $\left(r_{n u+i}^{*}, c_{n u+j}^{*}, e_{n u+k}^{*}\right)$, which we take with the opposite orientation, in a unique embedding $\psi_{i, j}$. Figure 1 illustrates the situation.

Finally, cut out from the supporting surfaces each such pair of corresponding triangles, one pair at a time, and identify the corresponding vertices $\left(x^{*}\right.$ with $\left.x\right)$ and edges bordering the two holes. After dealing with all $m n^{2}$ pairs of corresponding triangles in this fashion the result, as proved in [6], is a face 2-colourable triangular embedding $\chi$ of $K_{m n, m n, m n}$. The vertex set of $\chi$ is $\bar{R} \cup \bar{C} \cup \bar{E}$, where $\bar{R}=\left\{r_{n u+i}: 0 \leqslant u \leqslant m-1,0 \leqslant i \leqslant n-1\right\}$, $\bar{C}=\left\{c_{n u+i}: 0 \leqslant u \leqslant m-1,0 \leqslant i \leqslant n-1\right\}$ and $\bar{E}=\left\{e_{n u+i}: 0 \leqslant u \leqslant m-1,0 \leqslant i \leqslant n-1\right\}$ are three disjoint sets.

We are now in a position to state our main result which reinterprets the above construction in terms of Latin squares. 


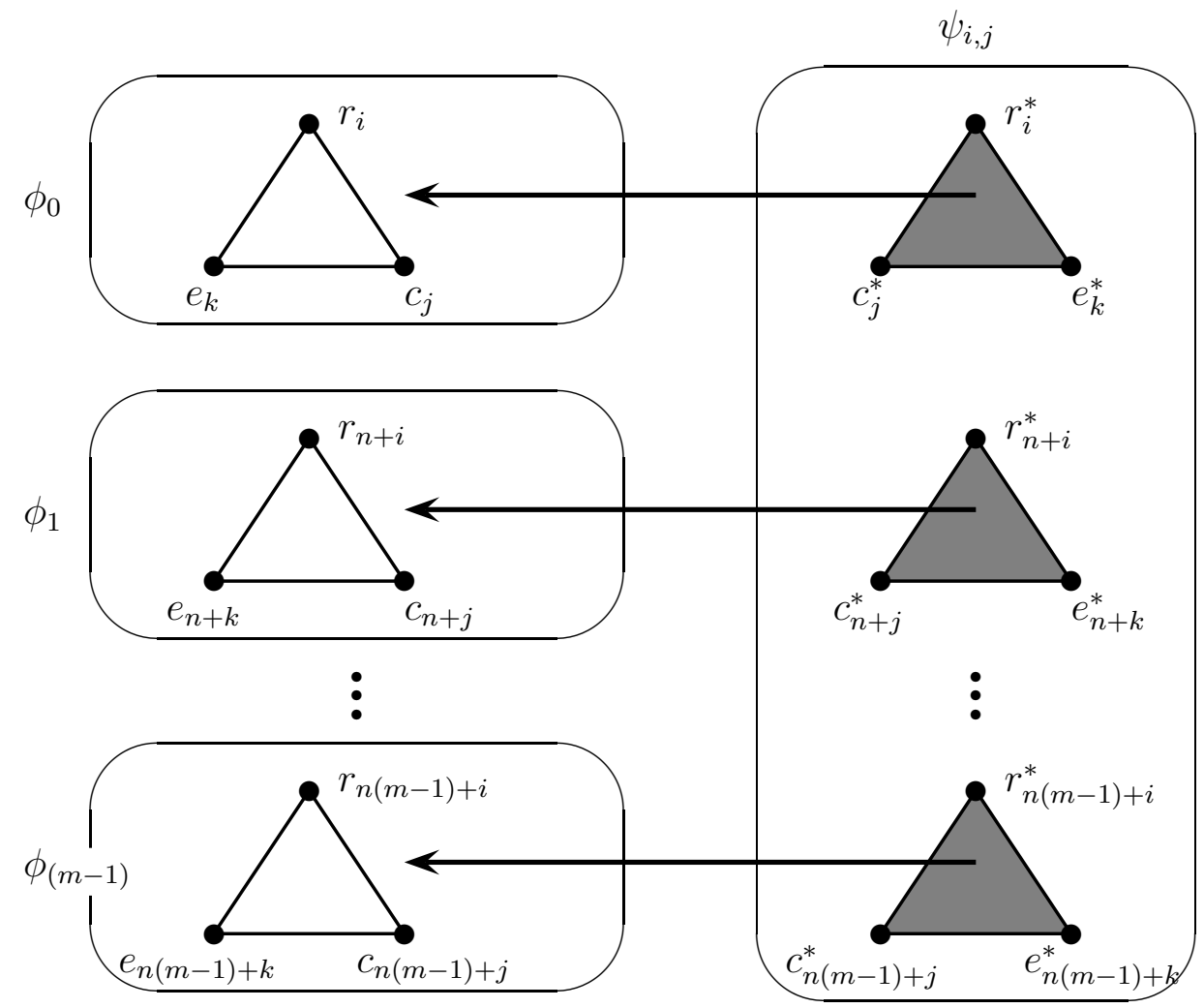

Each $\phi_{u}$ is an embedding of $K_{n, n, n}$ and $\psi_{i, j}$ is an embedding of $K_{m, m, m}$.

Figure 1. The construction.

Theorem 2.1 Suppose that, for $0 \leqslant u \leqslant m-1, L \bowtie L_{u}^{\prime}$, where $L$ and each $L_{u}^{\prime}$ are of order $n$ and have row, column and entry labels $\{0,1, \ldots, n-1\}$. Suppose also that for each $(i, j)$ with $0 \leqslant i, j \leqslant n-1, Q_{i, j} \bowtie Q_{i, j}^{\prime}$, where both $Q_{i, j}$ and $Q_{i, j}^{\prime}$ are of order $m$, and have row, column and entry labels $\{0,1, \ldots, m-1\}$, and that the squares $Q_{i, j}^{\prime}$ have a common transversal $\mathcal{T}$. Define $A$ and $A^{\prime}$, Latin squares of order mn with row, column and entry labels $\{0,1, \ldots, m n-1\}$, by

$$
\begin{aligned}
A(n u+i, n v+j) & =n Q_{i, j}(u, v)+L(i, j), \\
A^{\prime}(n u+i, n v+j) & =n Q_{i, j}^{\prime}(u, v)+k
\end{aligned}
$$

where $k= \begin{cases}L(i, j) & \text { if }(u, v, w) \notin \mathcal{T} \text { for any } w \\ L_{u}^{\prime}(i, j) & \text { if there exists } w \text { such that }(u, v, w) \in \mathcal{T},\end{cases}$

for $0 \leqslant u, v \leqslant m-1$ and $0 \leqslant i, j \leqslant n-1$. Then $A \bowtie A^{\prime}$.

Proof. Throughout the proof and subsequent discussions, we take the triangles determined by $L, Q_{i, j}$ and $A$ to be white, and those determined by $L_{u}^{\prime}, Q_{i, j}^{\prime}$ and $A^{\prime}$ to be black. Note that the biembeddings $L \bowtie L_{u}^{\prime}$ all have the same set of white triangles. First, for each $u$, 
we relabel the two Latin squares of the biembedding $L \bowtie L_{u}^{\prime}$ by adding $n u$ to each of the row, column and entry labels. This converts them to the range $\{n u, n u+1, n u+2, \ldots, n u+$ $n-1\}$. The resulting $m$ embeddings of $K_{n, n, n}$ may then be represented on $m$ disjoint surfaces, and the vertex set of the $u^{\text {th }}$ embedding $\phi_{u}$ may be taken as $R_{u} \cup C_{u} \cup E_{u}$, where $R_{u}=\left\{r_{n u+i}: 0 \leqslant i \leqslant n-1\right\}, C_{u}=\left\{c_{n u+i}: 0 \leqslant i \leqslant n-1\right\}$ and $E_{u}=\left\{e_{n u+i}: 0 \leqslant i \leqslant n-1\right\}$ are three disjoint sets representing the rows, columns and entries of the relabelled Latin squares. Thus the triple $\left(r_{n u+i}, c_{n u+j}, e_{n u+k}\right)$ defines a white triangle of $\phi_{u}$ if and only if $k=L(i, j)$, and it defines a black triangle of $\phi_{u}$ if and only if $k=L_{u}^{\prime}(i, j)$.

Next suppose that $\mathcal{T}=\left\{\left(\alpha_{u}, \beta_{u}, \gamma_{u}\right): 0 \leqslant u \leqslant m-1\right\}$ where, for each $u, \gamma_{u}=$ $Q_{i, j}^{\prime}\left(\alpha_{u}, \beta_{u}\right)$ for every $(i, j)$. Note that $\left\{\alpha_{u}: 0 \leqslant u \leqslant m-1\right\}=\{0,1, \ldots, m-1\}$, and similarly for $\beta$ and $\gamma$. Also, without loss of generality, we may take $\alpha_{u}=u$ for each $u$. For each of the $n^{2}$ pairs $(i, j)$, we relabel the two Latin squares $Q_{i, j}$ and $Q_{i, j}^{\prime}$ using the white triangle $(i, j, k)$ defined by $k=L(i, j)$ and the transversal $\mathcal{T}$; each row label $\alpha_{u}$ is renamed as $n u+i$, each column label $\beta_{u}$ is renamed as $n u+j$, and each entry label $\gamma_{u}$ is renamed as $n u+k$. The resulting $n^{2}$ embeddings of $K_{m, m, m}$ may then be represented on $n^{2}$ disjoint surfaces, which we will take to be disjoint from those of the biembeddings $\phi_{u}$. The vertex set of the $(i, j)^{\text {th }}$ embedding $\psi_{i, j}$ may be taken as $R_{i, j}^{*} \cup C_{i, j}^{*} \cup E_{i, j}^{*}$, where $R_{i, j}^{*}=\left\{r_{n u+i}^{*}: 0 \leqslant u \leqslant m-1\right\}, C_{i, j}^{*}=\left\{c_{n u+j}^{*}: 0 \leqslant u \leqslant m-1\right\}$ and $E_{i, j}^{*}=\left\{e_{n u+k}^{*}: 0 \leqslant u \leqslant m-1\right\}$ are three disjoint sets representing the rows, columns and entries of the relabelled Latin square. Thus the triple $\left(r_{n u+i}^{*}, c_{n v+j}^{*}, e_{n w+k}^{*}\right)$ defines a white triangle of $\psi_{i, j}$ if and only if $k=L(i, j)$ and $\gamma_{w}=Q_{i, j}\left(\alpha_{u}, \beta_{v}\right)$, and it defines a black triangle of $\psi_{i, j}$ if and only if $k=L(i, j)$ and $\gamma_{w}=Q_{i, j}^{\prime}\left(\alpha_{u}, \beta_{v}\right)$. Note that if $k=L(i, j)$ then $\left(r_{n u+i}^{*}, c_{n u+j}^{*}, e_{n u+k}^{*}\right)$ is a black triangle of $\psi_{i, j}$ for each $u \in\{0,1, \ldots, m-1\}$.

With the relabellings described in the previous two paragraphs, the biembeddings $\phi_{l}$ and $\psi_{i, j}$ correspond precisely to the construction from [6] as described at the start of this section. By cutting out from the supporting surfaces each pair of corresponding triangles, and identifying the corresponding vertices and edges, the result is a face 2colourable triangular embedding $\chi$ of $K_{m n, m n, m n}$. Our labelling of the points gives this embedding on the vertex set $\bar{R} \cup \bar{C} \cup \bar{E}$, where $\bar{R}=\left\{r_{n u+i}: 0 \leqslant u \leqslant m-1,0 \leqslant i \leqslant n-1\right\}$, $\bar{C}=\left\{c_{n u+i}: 0 \leqslant u \leqslant m-1,0 \leqslant i \leqslant n-1\right\}$ and $\bar{E}=\left\{e_{n u+i}: 0 \leqslant u \leqslant m-1,0 \leqslant i \leqslant n-1\right\}$ are three disjoint sets. We next identify for $\chi$ the two Latin squares $B$ (white) and $B^{\prime}$ (black) for which the biembedding $B \bowtie B^{\prime}$ gives $\chi$.

Take first a typical white triangle of $\chi$ having the edge $\left\{r_{n u+i}, c_{n v+j}\right\}$. This triangle comes from the embedding $\psi_{i, j}$, and so the third vertex is $e_{n w+k}$ where $k=L(i, j)$ and $w$ is given by $\gamma_{w}=Q_{i, j}\left(\alpha_{u}, \beta_{v}\right)$. Thus the Latin square $B$ giving the white triangles of $\chi$ is represented with row, column and entry labels $\{0,1, \ldots, m n-1\}$ by

$$
B(n u+i, n v+j)=n w+k, \text { where } \gamma_{w}=Q_{i, j}\left(\alpha_{u}, \beta_{v}\right) \text { and } k=L(i, j) .
$$

Black triangles of $\chi$ are of two types: those from the embeddings $\phi_{u}$, and those from the embeddings $\psi_{i, j}$. The former have an edge $\left\{r_{n u+i}, c_{n u+j}\right\}$ and then the third vertex is $e_{n u+k}$ where $k=L_{u}^{\prime}(i, j)$. The latter have an edge $\left\{r_{n u+i}, c_{n v+j}\right\}$ where $v \neq u$, and the third vertex is $e_{n w+k}$ where $k=L(i, j)$ and $w$ is given by $\gamma_{w}=Q_{i, j}^{\prime}\left(\alpha_{u}, \beta_{v}\right)$. Thus the Latin square $B^{\prime}$ giving the black triangles of $\chi$ is represented with row, column and entry 
labels $\{0,1, \ldots, m n-1\}$ by

$$
B^{\prime}(n u+i, n v+j)=n w+k, \text { where } \gamma_{w}=Q_{i, j}^{\prime}\left(\alpha_{u}, \beta_{v}\right) \text { and } k= \begin{cases}L(i, j) & \text { if } v \neq u, \\ L_{u}^{\prime}(i, j) & \text { if } v=u .\end{cases}
$$

Our final step is to permute the row, column and entry labels of $B$ and $B^{\prime}$ by defining

$$
\begin{aligned}
& A\left(n \alpha_{u}+i, n \beta_{v}+j\right)=n \gamma_{w}+k \Leftrightarrow B(n u+i, n v+j)=n w+k, \\
& A^{\prime}\left(n \alpha_{u}+i, n \beta_{v}+j\right)=n \gamma_{w}+k \Leftrightarrow B^{\prime}(n u+i, n v+j)=n w+k \text {, }
\end{aligned}
$$

where $u, v, w \in\{0,1, \ldots, m-1\}$ and $i, j, k \in\{0,1, \ldots, n-1\}$. Since $B \bowtie B^{\prime}$, we have $A \bowtie A^{\prime}$. Furthermore, and noting that we have taken $\alpha_{u}=u$ so that $L_{\alpha_{u}}^{\prime}=L_{u}^{\prime}$,

$$
\begin{aligned}
A(n u+i, n v+j) & =n Q_{i, j}(u, v)+L(i, j), \\
A^{\prime}(n u+i, n v+j) & =n Q_{i, j}^{\prime}(u, v)+k,
\end{aligned}
$$

where $k= \begin{cases}L(i, j) & \text { if }(u, v, w) \notin \mathcal{T} \text { for any } w, \\ L_{u}^{\prime}(i, j) & \text { if there exists } w \text { such that }(u, v, w) \in \mathcal{T},\end{cases}$

for $0 \leqslant u, v \leqslant m-1$ and $0 \leqslant i, j \leqslant n-1$. This completes the proof.

To illustrate Theorem 2.1 we give three examples which differ very slightly. All three take $m=n=3$ and make use of the following Latin squares.

$$
M=\begin{array}{l|lll} 
& 0 & 1 & 2 \\
\hline 0 & 0 & 1 & 2 \\
1 & 1 & 2 & 0 \\
2 & 2 & 0 & 1
\end{array} \quad N=\begin{array}{r|lll} 
& 0 & 1 & 2 \\
\hline 0 & 1 & 2 & 0 \\
1 & 2 & 0 & 1 \\
2 & 0 & 1 & 2
\end{array} \quad \quad O=\begin{array}{r|lll|} 
& 0 & 1 & 2 \\
\hline 0 & 2 & 0 & 1 \\
1 & 0 & 1 & 2 \\
2 & 1 & 2 & 0 \\
\hline
\end{array}
$$

Note that $M \bowtie N, M \bowtie O$ and $O \bowtie N$. In all three examples we take $Q_{i, j}^{\prime}=N$ for all $i$ and $j$, and we take $\mathcal{T}$ to be the transversal highlighted in $N$. In Theorem 2.1 it suffices that the squares $Q_{i, j}^{\prime}$ have a common transversal, but in our examples these squares are identical.

Example 2.1 In this example take $L=M, L_{0}^{\prime}=L_{1}^{\prime}=L_{2}^{\prime}=N, Q_{i, j}=M$ and $Q_{i, j}^{\prime}=N$, $0 \leqslant i, j \leqslant 2$. The entries of $A^{\prime}$ which arise from the transversal $\mathcal{T}$ are highlighted.

$A=$\begin{tabular}{c|ccccccccc}
\hline & 1 & 2 & 3 & 4 & 5 & 6 & 7 & 8 \\
\hline 0 & 0 & 1 & 2 & 3 & 4 & 5 & 6 & 7 & 8 \\
1 & 1 & 2 & 0 & 4 & 5 & 3 & 7 & 8 & 6 \\
2 & 2 & 0 & 1 & 5 & 3 & 4 & 8 & 6 & 7 \\
3 & 3 & 4 & 5 & 6 & 7 & 8 & 0 & 1 & 2 \\
4 & 4 & 5 & 3 & 7 & 8 & 6 & 1 & 2 & 0 \\
5 & 5 & 3 & 4 & 8 & 6 & 7 & 2 & 0 & 1 \\
6 & 6 & 7 & 8 & 0 & 1 & 2 & 3 & 4 & 5 \\
7 & 7 & 8 & 6 & 1 & 2 & 0 & 4 & 5 & 3 \\
8 & 8 & 6 & 7 & 2 & 0 & 1 & 5 & 3 & 4
\end{tabular}$\quad \quad A^{\prime}=\quad$\begin{tabular}{r|lllllllll|}
3 \\
\hline
\end{tabular}


By computing the rotation scheme one can check that $A \bowtie A^{\prime}$. For example, the rotation at $r_{4}$ is

$$
r_{4}: c_{0} e_{4} c_{8} e_{0} c_{5} e_{6} c_{2} e_{3} c_{7} e_{2} c_{4} e_{8} c_{1} e_{5} c_{6} e_{1} c_{3} e_{7}
$$

Example 2.2 Now take $L=M, L_{0}^{\prime}=L_{2}^{\prime}=N, L_{1}^{\prime}=O, Q_{i, j}=M$ and $Q_{i, j}^{\prime}=N$, $0 \leqslant i, j \leqslant 2$. We highlight those entries of $A$ and $A^{\prime}$ which correspond to $\phi_{1}: L \bowtie L_{1}^{\prime}=$ $M \bowtie O$.

$A=$\begin{tabular}{c|ccccccccc} 
& 0 & 1 & 2 & 3 & 4 & 5 & 6 & 7 & 8 \\
\hline 0 & 0 & 1 & 2 & 3 & 4 & 5 & 6 & 7 & 8 \\
1 & 1 & 2 & 0 & 4 & 5 & 3 & 7 & 8 & 6 \\
2 & 2 & 0 & 1 & 5 & 3 & 4 & 8 & 6 & 7 \\
3 & 3 & 4 & 5 & 6 & 7 & 8 & 0 & 1 & 2 \\
4 & 4 & 5 & 3 & 7 & 8 & 6 & 1 & 2 & 0 \\
5 & 5 & 3 & 4 & 8 & 6 & 7 & 2 & 0 & 1 \\
6 & 6 & 7 & 8 & 0 & 1 & 2 & 3 & 4 & 5 \\
7 & 7 & 8 & 6 & 1 & 2 & 0 & 4 & 5 & 3 \\
8 & 8 & 6 & 7 & 2 & 0 & 1 & 5 & 3 & 4
\end{tabular}$\quad \quad A^{\prime}=\quad$\begin{tabular}{r|lllllllll|}
3 \\
\hline
\end{tabular}

As in the first example, we present the rotation at $r_{4}$,

$r_{4}: c_{0} e_{4} c_{7} e_{2} c_{4} e_{8} c_{1} e_{5} c_{8} e_{0} c_{5} e_{6} c_{2} e_{3} c_{6} e_{1} c_{3} e_{7}$

Example 2.3 In this example take $L=M, L_{0}^{\prime}=L_{1}^{\prime}=L_{2}^{\prime}=N, Q_{i, j}=M$ for $0 \leqslant i, j \leqslant 2$ and $(i, j) \neq(1,2), Q_{1,2}=O$ and $Q_{i, j}^{\prime}=N$ for $0 \leqslant i, j \leqslant 2$. We highlight those entries of $A$ and $A^{\prime}$ which correspond to $\psi_{1,2}: Q_{1,2} \bowtie Q_{1,2}^{\prime}=O \bowtie N$.

$A=$\begin{tabular}{c|ccccccccc} 
& 0 & 1 & 2 & 3 & 4 & 5 & 6 & 7 & 8 \\
\hline 0 & 0 & 1 & 2 & 3 & 4 & 5 & 6 & 7 & 8 \\
1 & 1 & 2 & 6 & 4 & 5 & 0 & 7 & 8 & 3 \\
2 & 2 & 0 & 1 & 5 & 3 & 4 & 8 & 6 & 7 \\
3 & 3 & 4 & 5 & 6 & 7 & 8 & 0 & 1 & 2 \\
4 & 4 & 5 & 0 & 7 & 8 & 3 & 1 & 2 & 6 \\
5 & 5 & 3 & 4 & 8 & 6 & 7 & 2 & 0 & 1 \\
6 & 6 & 7 & 8 & 0 & 1 & 2 & 3 & 4 & 5 \\
7 & 7 & 8 & 3 & 1 & 2 & 6 & 4 & 5 & 0 \\
8 & 8 & 6 & 7 & 2 & 0 & 1 & 5 & 3 & 4
\end{tabular}$\quad \quad A^{\prime}=\quad$\begin{tabular}{r|lllllllll|}
3 \\
\hline
\end{tabular}

Again we present the rotation at $r_{4}$,

\section{$r_{4}: c_{0} e_{4} c_{8} e_{6} c_{2} e_{0} c_{5} e_{3} c_{7} e_{2} c_{4} e_{8} c_{1} e_{5} c_{6} e_{1} c_{3} e_{7}$}

Corollary 2.1.1 below gives a simplified version of Theorem 2.1, obtained by taking $L_{u}^{\prime}$ to be independent of $u$, and $Q_{i, j}^{\prime}$ to be independent of $(i, j)$. It also introduces a notation for the resulting squares $A$ and $A^{\prime}$ to emphasize their dependency on $L, L^{\prime}, Q$ and $Q^{\prime}$. The corollary and the notation will be useful in the subsequent section. 
Corollary 2.1.1 Suppose that $L \bowtie L^{\prime}$, where $L$ and $L^{\prime}$ are of order $n$ and have row, column and entry labels $\{0,1, \ldots, n-1\}$. Suppose also that $Q \bowtie Q^{\prime}$, where $Q$ and $Q^{\prime}$ are of order $m$ and have row, column and entry labels $\{0,1, \ldots, m-1\}$, and that the square $Q^{\prime}$ has a transversal $\mathcal{T}$. Define Latin squares $Q(L)$ and $Q^{\prime}\left(L, \mathcal{T}, L^{\prime}\right)$, Latin squares of order mn with row, column and entry labels $\{0,1, \ldots, m n-1\}$, by

$$
\begin{gathered}
Q(L)(n u+i, n v+j)=n Q(u, v)+L(i, j), \\
Q^{\prime}\left(L, \mathcal{T}, L^{\prime}\right)(n u+i, n v+j)=n Q^{\prime}(u, v)+k, \\
\text { where } k= \begin{cases}L(i, j) & \text { if }(u, v, w) \notin \mathcal{T} \text { for any } w, \\
L^{\prime}(i, j) & \text { if there exists } w \text { such that }(u, v, w) \in \mathcal{T},\end{cases}
\end{gathered}
$$

for $0 \leqslant u, v \leqslant m-1$ and $0 \leqslant i, j \leqslant n-1$. Then $Q(L) \bowtie Q^{\prime}\left(L, \mathcal{T}, L^{\prime}\right)$.

The square $Q(L)$ is partitioned into $n \times n$ subsquares which are just relabelled copies of $L$. The square $Q^{\prime}\left(L, \mathcal{T}, L^{\prime}\right)$ has a similar structure but the subsquares corresponding to the transversal $\mathcal{T}$ are relabelled copies of $L^{\prime}$. Note that if $L^{\prime}$ has a transversal, then among the relabelled copies of $L^{\prime}$ one can find a transversal in $Q^{\prime}\left(L, \mathcal{T}, L^{\prime}\right)$. This feature facilitates re-application of the construction and can be illustrated by reference to Example 2.1 which represents $M(M) \bowtie N(M, \mathcal{T}, N)$, where $\mathcal{T}$ is the highlighted transversal of $N$. Because $N$ has a transversal, $N(M, \mathcal{T}, N)$ has a transversal $\mathcal{U}$ within the highlighted cells. This transversal is given by $\mathcal{U}=\{(0,4,8),(1,5,7),(2,3,6),(3,7,5),(4,8,4),(5,6,3),(6,1,2)$, $(7,2,1),(8,0,0)\}$.

\section{Applications}

We begin this section by observing that if $Q$ and $R$ are Cayley tables of groups $\mathcal{Q}$ and $\mathcal{R}$ represented respectively on $\{0,1, \ldots, m-1\}$ and $\{0,1, \ldots, n-1\}$, then $Q(R)$ as defined in Section 2 is the Cayley table of the group $\mathcal{Q} \times \mathcal{R}$ represented on $\{0,1, \ldots, m n-1\}$. Thus, if $Q$ is taken as the Cayley table of $C_{2}$, then the square $Q(Q)$ gives the Cayley table for $C_{2}^{2}$. Repeating the process we see that the Cayley table for the elementary Abelian 2 -group $C_{2}^{k}$ is $Q(Q(\ldots(Q) \ldots))$, where there are $k$ occurrences of the symbol $Q$. Based on this observation we can prove the following theorem where, from now onwards, we identify each group with its Cayley table.

Theorem 3.1 For every $k, k \neq 2$, there is a Latin square $A_{k}^{\prime}$ such that $C_{2}^{k} \bowtie A_{k}^{\prime}$. Moreover, if $k>2$ then the square $A_{k}^{\prime}$ may be taken to contain a transversal. For $k=2$ there is no $A_{2}^{\prime}$ such that $C_{2}^{2} \bowtie A_{2}^{\prime}$.

$$
C_{2}=\begin{array}{l|ll} 
& 0 & 1 \\
\hline 0 & 0 & 1 \\
1 & 1 & 0
\end{array} \mid \quad A_{1}^{\prime}=\begin{array}{l|ll|} 
& 0 & 1 \\
\hline 0 & 1 & 0 \\
1 & 0 & 1 \\
\hline
\end{array}
$$

Table 1. The squares $C_{2}$ and $A_{1}^{\prime}$ forming a biembedding. 
Proof. For $k=1$, Table 1 gives squares $A=C_{2}$ and $A_{1}^{\prime}$ which provide the biembedding; this is clearly unique up to isomorphism and neither square has a transversal.

For $k=2$, it was shown in [3] that there is no biembedding of $C_{2}^{2}$ with any Latin square. For $k=3$, it was shown in [4] that there are 49 nonisomorphic biembeddings in which one of the squares is $C_{2}^{3}$. Amongst these 49 , the one with the largest automorphism group (of order 48) is the biembedding shown in Table 2.

$$
\begin{array}{c|cccccccc} 
& 0 & 1 & 2 & 3 & 4 & 5 & 6 & 7 \\
\hline 0 & 0 & 1 & 2 & 3 & 4 & 5 & 6 & 7 \\
1 & 1 & 0 & 3 & 2 & 5 & 4 & 7 & 6 \\
2 & 2 & 3 & 0 & 1 & 6 & 7 & 4 & 5 \\
3 & 3 & 2 & 1 & 0 & 7 & 6 & 5 & 4 \\
4 & 4 & 5 & 6 & 7 & 0 & 1 & 2 & 3 \\
5 & 5 & 4 & 7 & 6 & 1 & 0 & 3 & 2 \\
6 & 6 & 7 & 4 & 5 & 2 & 3 & 0 & 1 \\
7 & 7 & 6 & 5 & 4 & 3 & 2 & 1 & 0
\end{array} \quad \quad A_{3}^{\prime}=\quad \begin{array}{r|llllllll}
0 \\
\hline
\end{array}
$$

Table 2. The squares $C_{2}^{3}$ and $A_{3}^{\prime}$ forming a biembedding.

A transversal $\mathcal{T}_{3}$ is highlighted in the square $A_{3}^{\prime}$. By applying Corollary 2.1.1 we may then obtain a biembedding of $C_{2}^{6}=C_{2}^{3}\left(C_{2}^{3}\right)$ with $A_{6}^{\prime}=A_{3}^{\prime}\left(C_{2}^{3}, \mathcal{T}_{3}, A_{3}^{\prime}\right)$, and the latter square itself has a transversal $\mathcal{T}_{6}$. By repeating this process, it is clear that for $n \geqslant 1$, $C_{2}^{3 n} \bowtie A_{3 n}^{\prime}$ for some Latin square $A_{3 n}^{\prime}$ that has a transversal. This establishes the result for $k \equiv 0(\bmod 3)$.

\begin{tabular}{c|cccccccccccccccc|} 
& 0 & 1 & 2 & 3 & 4 & 5 & 6 & 7 & 8 & 9 & 10 & 11 & 12 & 13 & 14 & 15 \\
\hline 0 & 4 & 5 & 8 & 9 & 12 & 13 & 14 & 15 & 1 & 0 & 2 & 3 & 6 & 7 & 10 & 11 \\
1 & 5 & 4 & 9 & 8 & 13 & 12 & 15 & 14 & 0 & 1 & 3 & 2 & 7 & 6 & 11 & 10 \\
2 & 8 & 9 & 4 & 5 & 14 & 15 & 12 & 13 & 2 & 3 & 0 & 1 & 10 & 11 & 7 & 6 \\
3 & 9 & 8 & 5 & 4 & 15 & 14 & 13 & 12 & 3 & 2 & 1 & 0 & 11 & 10 & 6 & 7 \\
4 & 14 & 15 & 2 & 3 & 9 & 8 & 10 & 11 & 4 & 5 & 6 & 7 & 12 & 13 & 0 & 1 \\
5 & 15 & 14 & 3 & 2 & 8 & 9 & 11 & 10 & 5 & 4 & 7 & 6 & 13 & 12 & 1 & 0 \\
$A_{4}^{\prime}=$ & 2 & 3 & 15 & 14 & 10 & 11 & 8 & 9 & 6 & 7 & 4 & 5 & 0 & 1 & 12 & 13 \\
7 & 3 & 2 & 14 & 15 & 11 & 10 & 9 & 8 & 7 & 6 & 5 & 4 & 1 & 0 & 13 & 12 \\
8 & 13 & 12 & 6 & 7 & 0 & 1 & 4 & 5 & 10 & 11 & 14 & 15 & 8 & 9 & 2 & 3 \\
9 & 12 & 13 & 7 & 6 & 1 & 0 & 5 & 4 & 11 & 10 & 15 & 14 & 9 & 8 & 3 & 2 \\
10 & 6 & 7 & 12 & 13 & 4 & 5 & 0 & 1 & 14 & 15 & 11 & 10 & 2 & 3 & 8 & 9 \\
11 & 7 & 6 & 13 & 12 & 5 & 4 & 1 & 0 & 15 & 14 & 10 & 11 & 3 & 2 & 9 & 8 \\
12 & 0 & 1 & 10 & 11 & 2 & 3 & 6 & 7 & 8 & 9 & 12 & 13 & 5 & 4 & 14 & 15 \\
13 & 1 & 0 & 11 & 10 & 3 & 2 & 7 & 6 & 9 & 8 & 13 & 12 & 4 & 5 & 15 & 14 \\
14 & 10 & 11 & 0 & 1 & 6 & 7 & 3 & 2 & 12 & 13 & 8 & 9 & 14 & 15 & 4 & 5 \\
15 & 11 & 10 & 1 & 0 & 7 & 6 & 2 & 3 & 13 & 12 & 9 & 8 & 15 & 14 & 5 & 4 \\
\hline
\end{tabular}

Table 3. The square $A_{4}^{\prime}=A_{3}^{\prime}\left(C_{2}, \mathcal{T}_{3}, A_{1}^{\prime}\right)$. 
Next we consider the case $k \equiv 1(\bmod 3)$. We have $C_{2}^{3}\left(C_{2}\right)=C_{2}^{4}$ and this biembeds with $A_{3}^{\prime}\left(C_{2}, \mathcal{T}_{3}, A_{1}^{\prime}\right)=A_{4}^{\prime}$ by Corollary 2.1.1. The square $A_{4}^{\prime}$ is given in Table 3 . Although there is no transversal in $A_{1}^{\prime}$, there is a transversal in $A_{4}^{\prime}$, which is highlighted. Let us denote this transversal by $\mathcal{T}_{4}$. Again, by applying Corollary 2.1.1, we obtain a biembedding of $C_{2}^{7}=C_{2}^{4}\left(C_{2}^{3}\right)$ with $A_{7}^{\prime}=A_{4}^{\prime}\left(C_{2}^{3}, \mathcal{T}_{4}, A_{3}^{\prime}\right)$, and the latter square itself has a transversal $\mathcal{T}_{7}$. By repeating this process, it is clear that for $n \geqslant 1, C_{2}^{3 n+1} \bowtie A_{3 n+1}^{\prime}$ for some Latin square $A_{3 n+1}^{\prime}$ that has a transversal. This establishes the result for $k \equiv 1(\bmod 3)$.

Finally consider the case $k \equiv 2(\bmod 3)$. We have $C_{2}^{4}\left(C_{2}\right)=C_{2}^{5}$ and this biembeds with $A_{4}^{\prime}\left(C_{2}, \mathcal{T}_{4}, A_{1}^{\prime}\right)=A_{5}^{\prime}$. The square $A_{5}^{\prime}$ is too big to present here, but its construction from $A_{4}^{\prime}$ is obvious. The following set of (row, column, entry) triples forms a transversal in $A_{5}^{\prime}$ which we denote by $\mathcal{T}_{5}$.

$\{(0,16,2),(1,13,28),(2,17,1),(3,6,17),(4,3,18),(5,14,27),(6,1,19)$,

$(7,15,24),(8,4,4),(9,0,29),(10,8,16),(11,12,23),(12,2,6),(13,18,15)$,

$(14,10,20),(15,19,12),(16,5,13),(17,7,14),(18,9,3),(19,11,0)$,

$(20,20,22),(21,22,21),(22,24,7),(23,26,5),(24,21,25),(25,23,26)$,

$(26,28,30),(27,25,8),(28,30,10),(29,27,31),(30,29,11),(31,31,9)\}$.

Then $C_{2}^{5}\left(C_{2}^{3}\right)=C_{2}^{8}$ biembeds with $A_{5}^{\prime}\left(C_{2}^{3}, \mathcal{T}_{5}, A_{3}^{\prime}\right)=A_{8}^{\prime}$ which has a transversal $\mathcal{T}_{8}$. By repeating this process, it is clear that for $n \geqslant 1, C_{2}^{3 n+2} \bowtie A_{3 n+2}^{\prime}$ for some Latin square $A_{3 n+2}^{\prime}$ that has a transversal. This establishes the result for $k \equiv 2(\bmod 3)$ and completes the proof.

We remark that the square $A_{k}^{\prime}$ of Theorem 3.1 is not a paratopic copy of $C_{2}^{k}$ except in the case $k=1$. In fact, for $k>1, C_{2}^{k}$ is not biembeddable with a copy of itself, see [8].

We next make a conjecture concerning embeddings of groups.

Conjecture 3.1 Suppose that $G$ is a direct product of a finite number of cyclic groups. Then $G \bowtie H$ for some Latin square $H$, with the exception of $G=C_{2}^{2}$ where there is no biembedding.

If the conjecture is true, then every Abelian group $G$, with the single exception of $C_{2}^{2}$, will biembed with some other Latin square. In support of the conjecture, we make some observations.

Theorem 3.2 If $t$ is a positive integer, then $C_{t} \bowtie C_{t}^{\prime}$, where $C_{t}^{\prime}$ is a paratopic copy of $C_{t}$. Moreover, if $t$ is odd then $C_{t}$ (and hence also $C_{t}^{\prime}$ ) has a transversal.

Proof. The result follows from the existence of the so-called regular embedding of $K_{t, t, t}$, where the Latin squares involved are copies of $C_{t}$, [3]. If $t$ is odd then one of the transversals is the set of triples (row, column, entry) $\mathcal{T}=\{(i, i, 2 i) ; 0 \leqslant i<t\}$ with arithmetic modulo $t$.

Corollary 3.2.1 Suppose that $G$ is a direct product of a finite number of cyclic groups of odd order, at most one cyclic group of even order, and at most one elementary Abelian 2-group $C_{2}^{n}$ with $n>2$. Then $G \bowtie H^{\prime}$ for some $H^{\prime}$. 
Proof. When $G_{0}$ is a finite direct product of cyclic groups of odd order, repeated application of Corollary 2.1.1 together with Theorem 3.2 gives $G_{0} \bowtie H_{0}^{\prime}$ for some $H_{0}^{\prime}$ having a transversal. A further application of Corollary 2.1.1 together with Theorem 3.1 will deal with products involving a factor $C_{2}^{n}$ with $n>2$, and will yield a biembedding $G_{1} \bowtie H_{1}^{\prime}$ where $H_{1}^{\prime}$ has a transversal. A final application of Corollary 2.1.1 together with the regular embedding of a cyclic group of even order will deal with products involving a single factor of this type.

Note that Corollary 3.2.1 does not guarantee that $H^{\prime}$ has a transversal, although in practice it may have. For example, if we consider the biembedding $M\left(C_{2}\right) \bowtie N\left(C_{2}, \mathcal{T}, A_{1}^{\prime}\right)$, where $M$ and $N$ are the squares of order 3 used in the examples of Section $2, \mathcal{T}$ is the highlighted transversal of $N$, and $A_{1}^{\prime}$ is the square of order 2 used in Theorem 3.1, then it is easy to see that $H^{\prime}=N\left(C_{2}, \mathcal{T}, A_{1}^{\prime}\right)$ has a transversal while $M\left(C_{2}\right)=C_{3} \times C_{2}$. Actually, this $H^{\prime}$ is a paratopic copy of the square numbered 6.2 in the standard listing of [1], and the biembedding is also described in [3]. A further example is given in [4], where it is shown that $\left(C_{2} \times C_{4}\right) \bowtie D_{4}^{\prime}$, where $D_{4}^{\prime}$ is a square paratopic with the Cayley table of the dihedral group $D_{4}$ of order 8 , and again this square does have a transversal. On the other hand, it was shown in [3] that the only biembedding of $C_{4}$ is with a copy of itself, and this has no transversal.

Finally we turn to lower bounds on the numbers of biembeddings. In [2] it was proved that for $n=3 p$, where $p=2^{s}$ and $s$ is sufficiently large, there are at least $n^{n^{2} / 288}$ nonisomorphic face 2-colourable triangular embeddings of $K_{n, n, n}$, each of which has a parallel class in one colour. The proof depends on the construction of a biembedding of a pair of Latin squares of side $p$, one of which has $p^{2}(p-2) / 8$ subsquares of side 2 , and the other has a transversal. However, Theorem 3.1 establishes that for $s>2, C_{2}^{s} \bowtie A_{s}^{\prime}$, where $A_{s}^{\prime}$ has a transversal and $C_{2}^{s}$ has $p^{2}(p-1) / 4$ subsquares of side 2 . By using this biembedding and employing the same argument as in [2], the result is easily improved to show that for $n=3 p$, where $p=2^{s}$ and $s$ is sufficiently large, there are at least $n^{n^{2} / 144}$ nonisomorphic face 2-colourable triangular embeddings of $K_{n, n, n}$, each of which has a parallel class in one colour. Then, using this estimate, it is possible to improve, again by a factor 2 in the exponent, the lower bounds given in [2] for the numbers of nonisomorphic face 2-colourable triangular embeddings of $K_{n}$ in nonorientable surfaces for values of $n$ lying in the various infinite classes described in that paper. As a specific example, we now have that for $n=9 \cdot 2^{s+1}+1$, there are at least $n^{n^{2}\left(\frac{1}{1296}-o(1)\right)}$ such embeddings of $K_{n}$.

\section{References}

[1] C. J. Colbourn and J. H. Dinitz (editors), The CRC Handbook of Combinatorial Designs, 2nd Edition, CRC Press, Boca Raton (2006).

[2] M. J. Grannell and T. S. Griggs, A lower bound for the number of triangular embeddings of some complete graphs and complete tripartite graphs, J. Combin. Theory, Ser. B 98 (2008), 637-650. 
[3] M. J. Grannell, T. S. Griggs and M. Knor, Biembeddings of Latin squares and Hamiltonian decompositions, Glasgow Math. J. 46 (2004), 443-457.

[4] M. J. Grannell, T. S. Griggs and M. Knor, Biembeddings of Latin squares of side 8, Quasigroups Related Systems 15 (2007), 273-278.

[5] M. J. Grannell, T. S. Griggs, M. Knor and J. Širáň, Triangulations of orientable surfaces by complete tripartite graphs, Discrete Math. 306 (2006), 600-606.

[6] M. J. Grannell, T. S. Griggs and J. Širáň, Recursive constructions for triangulations, J. Graph Theory 39 (2002), 87-107.

[7] J. L. Gross and T. W. Tucker, Topological Graph Theory, John Wiley, New York (1987).

[8] J. G. Lefevre, D. M. Donovan, M. J. Grannell and T.S. Griggs, A constraint on the biembedding of Latin squares, Europ. J. Combin. 30 (2009), 380-386.

[9] G. Ringel, Map color theorem, Springer-Verlag, New York and Berlin (1974). 